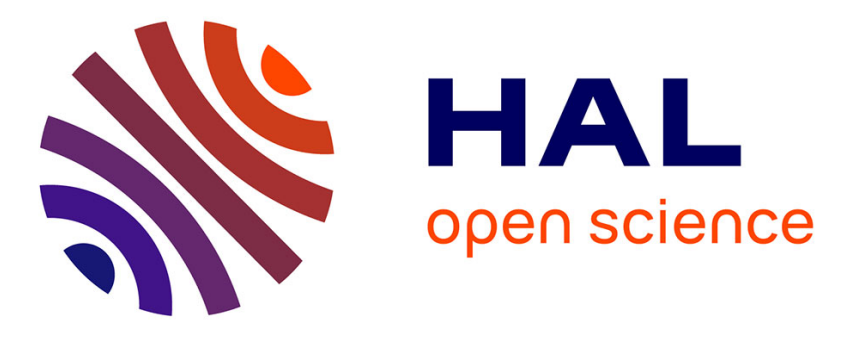

\title{
Revisiting neighbor discovery with interferences consideration
}

\author{
Elyes Ben Hamida, Guillaume Chelius, Eric Fleury
}

\section{To cite this version:}

Elyes Ben Hamida, Guillaume Chelius, Eric Fleury. Revisiting neighbor discovery with interferences consideration. 3rd ACM international workshop on Performance Evaluation of Wireless Ad hoc, Sensor and Ubiquitous Networks (PEWASUN 2006), ACM, Oct 2006, Terromolinos, Spain. pp.74-81, 10.1145/1163610.1163623 . inria-00384837

\section{HAL Id: inria-00384837 https://hal.inria.fr/inria-00384837}

Submitted on 15 May 2009

HAL is a multi-disciplinary open access archive for the deposit and dissemination of scientific research documents, whether they are published or not. The documents may come from teaching and research institutions in France or abroad, or from public or private research centers.
L'archive ouverte pluridisciplinaire HAL, est destinée au dépôt et à la diffusion de documents scientifiques de niveau recherche, publiés ou non, émanant des établissements d'enseignement et de recherche français ou étrangers, des laboratoires publics ou privés. 


\section{Revisiting Neighbor Discovery with Interferences Consideration}

\author{
Elyes Ben Hamida \\ CITI/ARES - INSA de Lyon \\ France \\ elyes.ben-hamida@insa- \\ lyon.fr
}

\author{
Guillaume Chelius \\ CITI/ARES - INRIA \\ France
}

guillaume.chelius@inria.fr

\author{
Eric Fleury \\ CITI/ARES - INSA de Lyon \\ France \\ eric.fleury@inria.fr
}

\begin{abstract}
In wireless multi-hop networks, hello protocols for neighbor discovery are a basic service offered by the networking stack. However, their study usually rely on rather simplistic models which do not take into account problems resulting from low level layers, such as the physical layer. One of the peculiarities of radio communications is the presence of interferences which decrease the capacity of the medium.

In this paper, we consider a random hello protocol inspired by aloha and we study the impact of the interferences on the neighbor discovery process. As expected, we prove that, in average and in the presence of interferences, a node discovers only a subset of its neighbors. We propose then an analytical model to compute the average number of nodes that a given node may expect to discover in its neighborhood.

Finally, we present a hello protocol with sleep periods. We show how to optimize this protocol using our hybrid model. A real scenario stemming from the CAPNET project is then analyzed and studied.
\end{abstract}

\section{Categories and Subject Descriptors}

C.2.1 [Computer Communication Networks]: Wireless communications

\section{General Terms}

Performance, Design

\section{Keywords}

Wireless multi-hop networks, Neighbor discovery, Stochastic geometry, Performance analysis

\section{INTRODUCTION}

\footnotetext{
*This work is partly supported by the European Commission, project AEOLUS IST-15964, and by the CAPNET project.
}

Wireless ad hoc and sensor networks are communication systems where the infrastructure is dynamically created and maintained. To enable communications, hosts cooperate together to provide several complex services like self-organization, routing or data gathering. All these high level services usually rely on a neighbor discovery protocol. During the process of neighbor discovery, a node tries to find out which other nodes are within its transmission range. To accomplish this discovery, a node broadcasts periodically a message (a.k.a. a a hello packet) to inform the nearby nodes of its presence. This periodic exchange of hello messages is used to create and maintain a local neighborhood table. This table is then used by higher level protocols to communicate with nearby nodes, for example to establish a route between two distant nodes.

Several studies were carried out on hello protocols. In [10], the impact of hello protocols in ad hoc networks is studied. In order to reduce the network congestion and increase the performances, three hello protocols are proposed. In [3], the AODV routing protocol is used to examine the effectiveness of hello messages for the monitoring of link status. Several factors influencing the utility of hello messages are determined, and a variety of approaches for improving the accuracy of these messages are evaluated. In [15], a differential hello technique is introduced to reduce the overhead induced by the hello messages. Besides these various studies, several works were done to propose and compare various types of random hello protocols [7-9,13].

In this paper, we are interested in the neighbor discovery process in presence of interferences. First, we study the impact of radio interferences on the process of neighbor discovery. Then, we propose a model allowing to estimate the average number of nodes which one can hope to discover in presence of interferences. Finally, we determine the optimal period of node activity that maximizes the number of discovered nodes and minimizes the energy consumption.

The remainder of this paper is organized as follows. In Section II, we review some of the related works on neighbor discovery in wireless networks. In Section III, we describe the models and assumptions. In Section IV, we show by simulations the impact of interferences on neighbor discovery. In Section V, we propose a hybrid model allowing to estimate the average number of nodes which we can hope to discover in presence of interferences. A hello protocol with sleep periods is presented and analyzed in section VI and we show how to use our model to tune this protocol in section VII. Conclusions and perspectives are finally given in Section VIII. 


\section{RELATED WORKS}

Several works have studied the design of hello protocols in the context of wireless multi-hop networks. McGlynn et al [13] propose a family of birthday protocols which use random transmissions to discover adjacent nodes in static ad hoc networks, where the nodes are supposed to be synchronized. The proposed mathematical models as well as the simulations show the energy efficiency and the robustness of such random protocols for neighbor discovery in comparison to deterministic or scheduling algorithms.

Alonso et al [8], provide a general model allowing to study and analyze hello protocols in ad hoc single broadcast channel networks. The time is slotted, the nodes are synchronized and they can be in one of the following two states: listening or talking. Using this model, the authors describe and compare various hello protocols. However, this model as well as the studied protocols do not take into account the energy consumption. In [7], Alonso et al adapt their model to the case of ad hoc multichannel broadcast networks.

Jakllari et al [9], propose a polling based MAC protocol that addresses the problem of neighbor discovery with directionnal antennas. This type of antenna increases the capacity of the network thanks to the spatial diversity. This protocol uses a polling strategy wherein a node polls its discovered neighbors periodically. This enable the node to adjust its antenna weighting coefficients in order to track its neighbors. The analytical study as well as the simulations show the efficiency of this protocol for mobile ad hoc networks in term of capacity enhancement.

Most of the studies made on hello protocols use rather simplistic models which do not take into account the specificities of radio communications. In our knowledge, few studies analyze the problem of neighbor discovery in presence of interferences.

\section{MODELS AND ASSUMPTIONS}

In this section, we present the various models and assumptions used in our study. First, we define the location model used to describe the position of nodes. Then, we present two sensing models for radio communication modeling. Finally, we describe the hello protocol used throughout our study.

\subsection{Location model}

We consider a large scale network with a large number of nodes dispatched in a vast two-dimensionnal geographical region. We assume that nodes are uniformly and independently distributed in the region.

Under this assumption, the nodes location can be modeled by a stationary two-dimensionnal Poisson point process of constant spatial intensity $\lambda$.

The number of nodes located in a region $A, B(A)$, follows a Poisson distribution of parameter $\lambda|A|$, where |.| represent the Lebesgue measure in $\mathbb{R}^{2}$. We have the following relation:

$$
\mathbb{P}[B(A)=k]=\frac{(\lambda|A|)^{k}}{k !} e^{-\lambda|A|}
$$

\subsection{Sensing models}

In this study, we consider two sensing models: a Boolean model and a more realistic one, called SINR model, which takes into account interferences. These two sensing models have been widely used in the literature $[5,12,14]$.

\subsubsection{Boolean sensing model}

Various models have been developed to model radio communications between nodes. One of these models, the Friis free space model [6], is widely used in the literature. Suppose there is a node $x$ that emits with constant power $P_{t}$. The node $y$, which is at the euclidean distance $d(x, y)$ of $x$, can receive the signal if and only if the received signal power, $P_{r}$, is above a given threshold, $\theta$. This model is given by:

$$
P_{r}=\frac{P_{t} K}{d(x, y)^{\beta}} \geq \theta
$$

where $K$ is a constant which depends on the antenna gain and the wavelength. $\beta$ is the path loss exponent. For radio signal sensing, the exponent typically ranges from 2.0 to 5.0. This model results into a perfect circular coverage area around each node with a maximal radius $R\left(P_{t}\right)$ defined as follows:

$$
R\left(P_{t}\right)=\left(\frac{P_{t} K}{\theta}\right)^{\frac{1}{\beta}}
$$

Thus, a node can only sense the environment and detect other nodes only within its maximal sensing area $R\left(P_{t}\right)$. A node is then said to be covered by another one if it lies within the node maximal sensing area. With this model, we propose a rather simple model for the management of interferences, which we call the Boolean model, where simultaneous communications of two or several nodes within reach communication, $R\left(P_{t}\right)$, yields to a collision.

\subsubsection{SINR sensing model}

The Boolean sensing model does not take into account correctly interferences stemming from radio communications of others nodes. A more accurate model based on the signal to interference ratio is presented in [11].

A node $x$ can receive a signal from a node $y$ if the ratio of power it receives from $y$ to the total power received from all other nodes is above a given threshold. This model is given by:

$$
\frac{\frac{P_{t} K}{d(x, y)^{\beta}}}{N+\gamma \sum_{k \neq(x, y)} \frac{P_{t} K}{d(k, y)^{\beta}}} \geq \theta
$$

where $N$ is the power of the thermal background noise and $\theta$ is the signal to noise ratio required for successful signal decoding. $\gamma$ depends on the orthogonality between the radio resources (codes or frequencies) used during simultaneous transmissions. In this study we suppose that $\gamma=1$.

With this more realistic model, we can analyze the impact of interferences on the neighbor discovery process.

\subsection{The random hello protocol}

The hello protocol that will be used throughout this study, is a random protocol inspired by Aloha [1]. Each node can be in one of the following two states: listening or talking. These two states occur inside a frame of duration $w$.



Figure 1: The random hello protocol. 
In every occurrence of this frame, a node picks randomly an instant $t_{i}$, such that $t_{i} \in[0, w-\delta]$. The hello message is then transmitted at $t_{i}$ with a duration of $\delta$. This transmission is made directly without any carrier sensing. Each node transmits only one hello message per frame and keeps listening to the medium during the remaining period of duration $w-\delta$. This protocol is depicted in figure 1 .

As for aloha, this random hello protocol presents a collision vulnerable zone of size $2 \times \delta$. So, given the Boolean sensing model, if two nodes which are within communication range pick two $t_{i}$ belonging to the same vulnerable zone, a collision occurs. Considering collisions in the Boolean sensing model, the probability of discovering a node is as follows:

$$
\mathbb{P}[\text { node discovery }]=\left(1-\frac{2 \delta}{w-\delta}\right)^{n-1}
$$

where $n-1$ is the number of nodes within the sensing area of the hello message transmitter. A node is assumed to be discovered when its hello packet is correctly received by other nodes (i.e. without collision).

\section{IMPACT OF INTERFERENCES ON NEIGH- BOR DISCOVERY}

In this section we study the impact of interferences on neighbor discovery. First, to show the impact of interferences, we present a graph of logical connectivity obtained after the simulation of the hello protocol with both sensing models that were presented in section 3 . Then, we measure the average degree of nodes according to the size of $w$. Finally, we study the impact of interferences on the average number and the average distance of the discovered nodes.

\subsection{Graph of connectivity}

During the process of neighbor discovery, when a node is discovered, its identity is added to the local neighborhood table of the nodes which have received the hello packet. From these tables, we can generate the corresponding graph of connectivity.

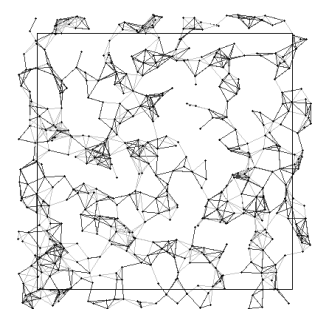

(a) Boolean model

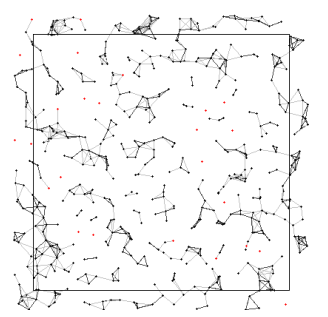

(b) SINR model
Figure 2: Graphs of connectivity for different sensing models. $\left(w=200, \delta=10, \lambda=0.0035, P_{t}=10^{5}\right.$, $\theta=5, \beta=3, N=1, K=1)$.

This notion of connectivity is logical as it is built upon the reception of packets as opposed to a physical connectivity which would only rely on the physical medium characteristics. In other terms, interferences and collisions affect the logical connectivity as they affect the correct reception of hello packets.

In figure 2(a), we present a graph of connectivity in the Boolean sensing model after one run of the hello protocol.
By one run, we mean that the hello protocol is executed only for a duration of $w$, with all nodes having their frame synchronized. In figure 2(b), a graph of connectivity in the SINR sensing model is also presented with the same parameters.

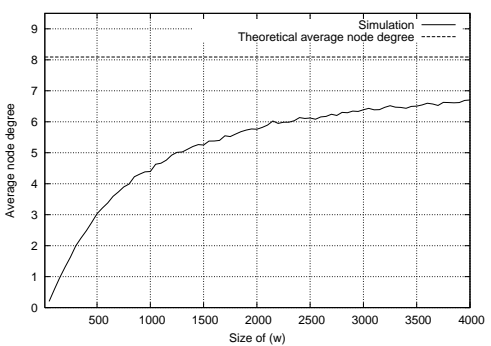

Figure 3: Node degree obtained after one run of the hello protocol.

Given that the SINR sensing model takes into account the interferences with more accuracy, we can observe that the number of discovered nodes is lower with regard to the Boolean model. The obtained graph is then disconnected with the existence of several isolated nodes. The more the frequency of hello messages is raised, the more the interferences increase. They are caused by simultaneous transmissions of several hello messages. To decrease these interferences, the parameter $w$ must be increased. This improve the probability of discovering a node.

As presented in the figure 3, the more the size of $w$ increases, the more the degree of nodes aims towards the theoretical value. This correlation enlightens the importance of an appropriate dimensioning of the protocol hello in order to maximize or to tune the number of discovered nodes during a given period.

\subsection{Impact of interferences on neighbor dis- covery}

In this section, we simulate the random hello protocol for various sizes of $w$ and for various numbers of run. For each size of $w$, we draw the graphs of connectivity corresponding to one and 10 runs of the hello protocol. The objective is to measure the number of nodes discovered according to the size of $w$ and the number of runs. As for each node and each run, the instant $t_{i}$ of hello packet transmission varies, one can expect to discover new neighbors at each run of the protocol.

The different graphs are shown in figure 4. First, for a given number of run, we can observe that a large value of $w$ yields to a decrease of the collision probability, and consequently, an increase in the number of discovered nodes. Besides, we notice that for a low $w$, the number of run does not influence the number of discovered nodes. This number remains lower than the total number of nodes which are present in the maximal sensing zone of radius $R\left(P_{t}\right)$ (see figure $4(\mathrm{~d})$ and section 3.2 .1 ). Only nodes which are close to the transmitter receive correctly the hello message. As interferences are high, the nodes can not discover all their theoretical neighborhood, given by the maximal sensing zone of radius $R\left(P_{t}\right)$, even if the protocol is executed during a long period. For larger values of $w$, the number of run does influence the number of discovered nodes. New nodes are dis- 


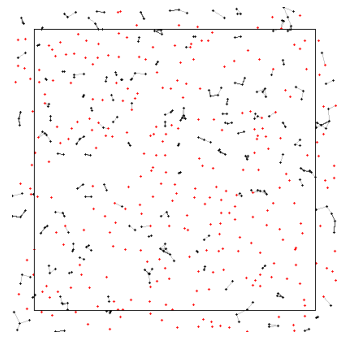

(a1) $\mathrm{w}=50$ (1 run)

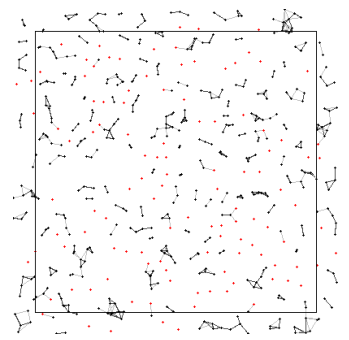

(a2) $\mathrm{w}=50$ (10 runs)

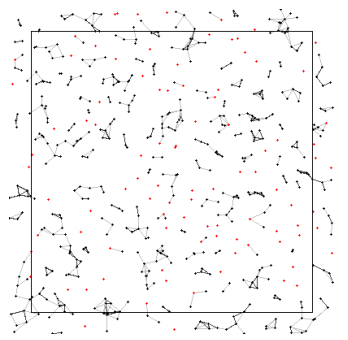

(b1) $\mathrm{w}=100$ (1 run)

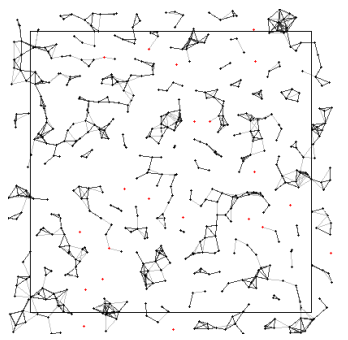

(b2) $\mathrm{w}=100$ (10 runs)

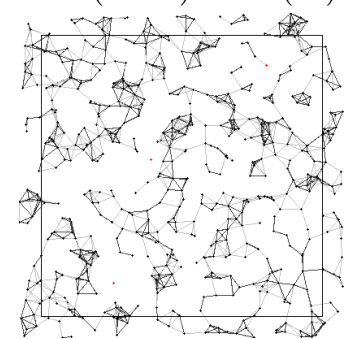

(c1) $\mathrm{w}=400$ (1 run)

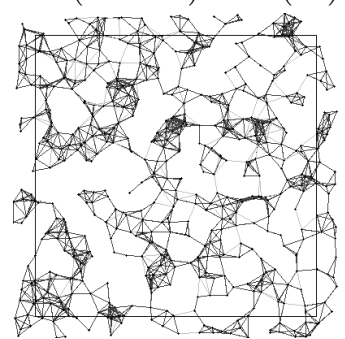

(c2 ) w=400 (10 runs)

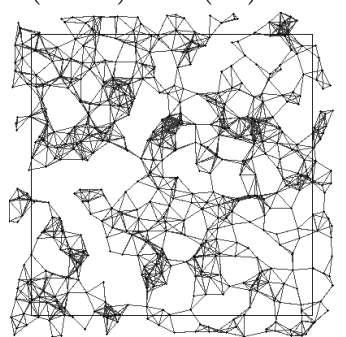

(d) Maximal sensing area $R\left(P_{t}\right)$

Figure 4: Impact of interferences on neighbor discovery. $\left(\delta=10, \lambda=0.0035, P_{t}=10^{5}, \theta=5, \beta=3, N=1\right.$, $K=1)$.

covered when the number of runs increase. However, even after a high number of runs, the zone where nodes are discovered remains smaller than the maximal sensing zone of radius $R\left(P_{t}\right)$.

This result suggests clearly that the number of discovered nodes depends on the frequency of hello messages transmission as well as interferences. There is thus a given upper bound, lower than the maximal sensing zone $R\left(P_{t}\right)$, and beyond which nodes are not discovered.

\section{A MODEL TO ESTIMATE THE AVER- AGE NUMBER OF DISCOVERED NODES}

In this section, we present a hybrid interference model which enables the computation of an upper-bound beyond which nodes are not discovered. With this upper-bound, we can then estimate the average number of nodes which one can hope to discover. Finally, we validate this model by simulations.

\subsection{Computation of the upper-bound}

Considering the SINR sensing model, when a node $x$ transmits a hello message, the node $y$ can decode this message only if the signal-to-noise ratio is above a given threshold $\theta$. From the equation 4 , and with $P_{t}, K, N$ and $\beta$ constant, the distance $d(x, y)$ has to satisfy the following condition:

$$
d(x, y) \leq\left(\frac{P_{t} K}{\theta(N+I)}\right)^{\frac{1}{\beta}}
$$

where $I$ is the shotnoise and represents the total power received from all other nodes. To compute the maximal value that $d(x, y)$ can take to satisfy this relation, it is necessary to estimate the value of $I$.

Several studies were realized to estimate the value of these interferences $[5,12,14]$. However, they did not result in a closed formula. In consequence, we propose a hybrid model to compute an estimate of $I$.
As shown in figure 5, consider a node $i$ and $R\left(P_{t}\right)$ its maximal sensing area in absence of interference as defined in section 3.2.1, i.e., such as: $R\left(P_{t}\right)=\left(\frac{P_{t} K}{\theta N}\right)^{\frac{1}{\beta}}$. We suppose an infinite plan around this node and we decompose the interferences in two parts: the internal interferences, $I_{i n}$, and the external interferences, $I_{\text {out }}$. $I_{\text {in }}$ corresponds to the interferences stemming from nodes localized inside the disc of centre $i$ and radius $R\left(P_{t}\right)$. $I_{\text {out }}$ corresponds to the interferences stemming from nodes which are located outside this disc.

Given that the nodes are distributed according to a stationary two-dimensionnal Poisson point process of constant spatial intensity $\lambda$, we can easily compute the average number of nodes present in a given area $S$. A direct implication of equation 1 is that the average number of nodes being in an area $S$ is equal to $\lambda S$. The average number of nodes localized at distance almost $r$ from a given node is equal to $2 \pi r \lambda$. So, to calculate $I_{\text {out }}$, we integrate the previous expression as follows:

$$
I_{\text {out }}=\int_{R\left(P_{t}\right)}^{+\infty}(2 \pi r \lambda \rho)\left(P_{t} r^{-\beta}\right) d r=\frac{2 \pi \lambda}{\beta-2} \rho P_{t} R\left(P_{t}\right)^{2-\beta}
$$

where:

- $\rho$ : is the medium access probability. In the case of our random hello protocol, this probability is equal to: $\rho=\frac{\delta}{w}$.

- $\beta$ : is the path loss exponent such that $\beta>2$. This condition on $\beta$ is imposed in order to ensure convergence of the integration, and this condition has also been imposed in all previous and related works (e.g. $[5,12,14])$.

To estimate the internal interferences, $I_{i n}$, is a much harder task. No closed formula is available. Furthermore, the integral that we consider diverges for small values of $r$. For this reason, we define a hybrid model which combines the SINR 
model for the external interferences and the Boolean model for the internal ones. In other words, given a transmitter $x$, we consider that a simultaneous transmission from a node $y$ such that $d(x, y) \leq R\left(P_{t}\right)$ leads necessarily to a collision and that both packets are lost. A simultaneous transmission from a node $y$ such that $d(x, y)>R\left(P_{t}\right)$ is added to the interferences computed in $I_{\text {out }}$.

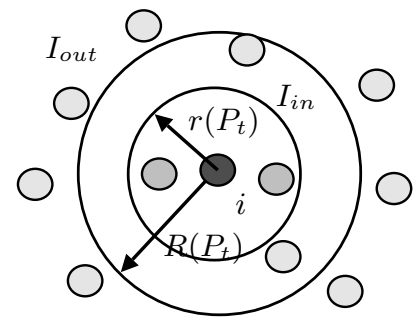

Figure 5: $r\left(P_{t}\right)$ vs $R\left(P_{t}\right)$.

As we only consider a subset of the interfering nodes, the global interferences are larger than $I_{\text {out }}$. The external interferences can be seen as being a lower-bound for the global interferences. By taking into account only these external interferences, we can compute an upper-bound on the distance $d(x, y)$ that verifies equation 6 as follows:

$$
d(x, y) \leq\left(\frac{P_{t} K}{\theta\left(N+\frac{2 \pi \lambda}{\beta-2} \rho P_{t} R\left(P_{t}\right)^{2-\beta}\right)}\right)^{\frac{1}{\beta}}
$$

In presence of interferences and according to the frequency of hello messages, the nodes which we can discover on average are those belonging to the $r\left(P_{t}\right)$ zone, where $P_{t}$ is the transmission power. $r\left(P_{t}\right)$ is defined as follows:

$$
r\left(P_{t}\right)=\left(\frac{P_{t} K}{\theta\left(N+\frac{2 \pi \lambda}{\beta-2} \rho P_{t} R\left(P_{t}\right)^{2-\beta}\right)}\right)^{\frac{1}{\beta}}
$$

In other words, in presence of interferences, each node discovers on average the nodes localized in the $r\left(P_{t}\right)$ zone at most, as shown in figure 5 . The other nodes belonging to the $\left[R\left(P_{t}\right)-r\left(P_{t}\right)\right]$ zone cannot be discovered. These results are valid only in average.

\subsection{Simulation results}

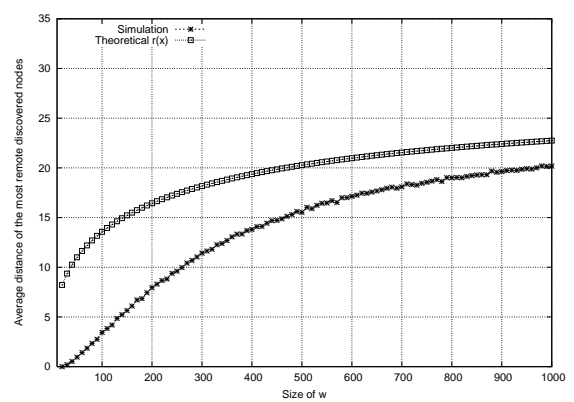

Figure 6: Average distance of the most remote discovered neighbor. $(\delta=10, \lambda=0.0035, \theta=5, \beta=3$, $N=1, K=1, P_{t}=10^{5}$ )

In this section, we verify the analytical value of $r\left(P_{t}\right)$ by simulations using the netsens [4] simulator. For that pur- pose, we simulate the random hello protocol for different values of $w$ and during one occurrence of $w$. For every $w$, we compute the average distance of the most remote neighbor who was discovered. We compare this simulation value with the analytical value of $r\left(P_{t}\right)$.

As shown in the figure 6 , the more $w$ increases, the more the average distance of the most remote discovered nodes aims towards $r\left(P_{t}\right)$. On the other hand, for low values of $w$ the internal interferences increase, and as a consequence the probability of collision also increases. In that case, the mean distance of the most remote neighbors is lower than $r\left(P_{t}\right)$.

Given our hybrid model, we are also able to tune $w$ in order to avoid internal interferences, i.e., to reduce the probability of collision between two nodes $x$ and $y$ such that $d(x, y) \leq R\left(P_{t}\right)$. With the knowledge of the external interferences, we know analytically $r\left(P_{t}\right)$ and thus, we know the average number of nodes we must consider for collisions in the Boolean model.

\section{A RANDOM HELLO PROTOCOL WITH SLEEP PERIODS}

In this section, we present a modified version of the random hello protocol. This new version includes sleep periods to reduce the energy consumption in each node. The impact of this sleep period on the performance of the neighborhood discovery is then analyzed.

\subsection{Description of the protocol}

As shown on figure 7 , each node can be in one of the following three states: listening, talking or sleeping. These three states are performed inside a frame, $F$, of size $w+s$.

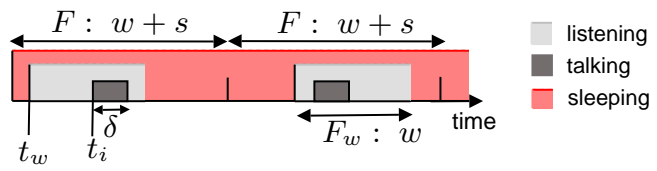

Figure 7: The random hello protocol with sleep period.

In each occurrence of $F$, a node picks randomly an instant $t_{w}$, such that $t_{w} \in[0, s]$. Then, a node picks randomly a second instant $t_{i}$, such that $t_{i} \in\left[t_{w}, t_{w}+w-\delta\right]$. A node is in the talking state during $\left[t_{i}, t_{i}+\delta\right]$, in the listening state during $\left[t_{w}, t_{w}+w\right] \backslash\left[t_{i}, t_{i}+\delta\right]$ and in the sleeping state the rest of the frame $F$. The hello message is transmitted at $t_{i}$ with a duration of $\delta$. In the sleep state, a node does not receive the messages.

In conclusion, for each occurrence of the frame $F$, a node transmits only one message with a duration of $\delta$, listens to the medium during $w-\delta$ and sleeps during $s$. The medium access probability is thus : $\rho=\frac{\delta}{w+s}$.

\subsection{Impact of the sleep period}

We notice from equation 9 that the more $s$ increases, the more the interferences decrease and the upper-bound $r\left(P_{t}\right)$ aims towards the maximal sensing zone, $R\left(P_{t}\right)$.

In figure 8 , the average node degree is shown for various values of $w$ and $s$. For each value of $w$, we notice that there is a particular value of $s$ which maximizes the average number of discovered nodes. 


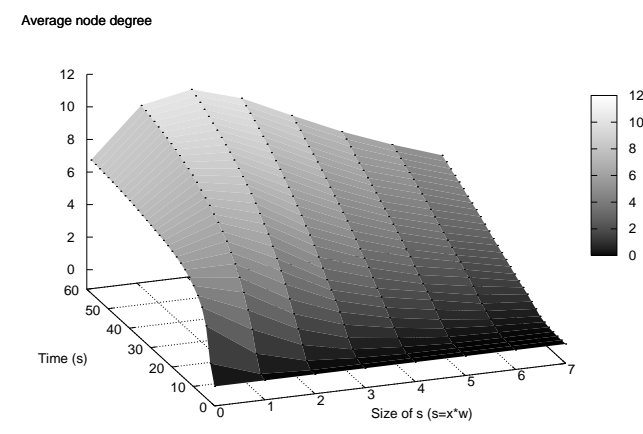

(a) $w=2000$

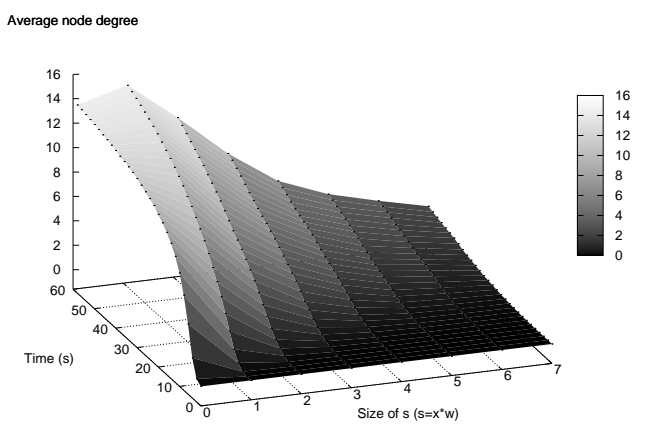

(b) $w=5000$

Figure 8: Average node degree for different $s$ and $w \cdot\left(\delta=10, \lambda=0.0035, P_{t}=5 \times 10^{5}, \theta=4, \beta=3, N=1, K=1\right.$, theoretical average node degree $\approx 27$ ).

We can state that the addition of a sleep period not only minimizes the energy consumption but also decreases interferences and thus may increase the performance of the hello protocol. On one hand, when the size of $s$ increases with regard to $w$, collisions decrease as well as interferences, improving the value of $r\left(P_{t}\right)$. On the other hand, some neighboring nodes may miss a hello packet and not discover a node because of the sleep period during which a node is not listening. This tradeoff is responsible for the existence of the optimal value of $s$ which is observed in the figure 8 . We can also observe that using a high value for $w$ increases the average number of discovered nodes but also s the energy consumption.

In conclusion, it seems that there is an optimal value for $s$ in regard to $w$, which minimizes the energy consumption and maximizes the average number of discovered nodes. A correct dimensioning of the hello protocol is then necessary to minimize the energy consumption, decrease the collisions and interferences and maximize the average number of nodes discovered during a given period of time.

\section{HELLO PROTOCOL DIMENSIONING}

In this section we present how to use our analytical results to tune the hello protocol. A real scenario stemming from the CAPNET project is then presented as a direct application.

\subsection{Dimensioning}

As an example of application for our analytical results, we propose to tune the hello protocol in order to achieve the following objectives:

- Discover all the nodes which are below a given distance $L$;

- Maximize the probability of discovering a node during a given period $T$;

- Minimize the energy consumption.

\subsubsection{Discovering all nodes below a distance $L$}

To discover the nodes which are below a distance $L$, such as $L \leq R\left(P_{t}\right)$, we have to satisfy the following relation:

$$
r\left(P_{t}\right)=\left(\frac{P_{t} K}{\theta\left(N+I_{\text {out }} \rho\right)}\right)^{\frac{1}{\beta}} \leq L
$$

where: $I_{\text {out }}=\frac{2 \pi \lambda}{\beta-2} P_{t} R\left(P_{t}\right)^{2-\beta}$, and $R\left(P_{t}\right)=\left(\frac{P_{t} K}{\theta N}\right)^{\frac{1}{\beta}}$.

For a constant transmission's power, the medium access probability, $\rho$, is then as follows:

$$
\rho \geq\left(\frac{P_{t} K}{\theta L^{\beta}}-N\right) \frac{1}{I_{\text {out }}}
$$

As $\rho=\frac{\delta}{w+s}$, thus, to discover all the nodes which are closer to a distance $L$, we have to configure our hello protocol with a frame, $F$, of size $w+s$, such that:

$$
w+s \leq \frac{\delta I_{\text {out }}}{\frac{P_{t} K}{\theta L^{\beta}}-N}
$$

\subsubsection{Probability of discovering a node}

Knowing the maximal size of $(w+s)$, we determine the size of $s$, that maximizes the probability of discovering a node during a frame $F$.

During one run of the hello protocol, the probability of discovering a node, $p$, is:

$$
p=\frac{w-\delta}{w+s}\left(1-\frac{2 \delta}{w+s}\right)^{n-2}
$$

where $n$ is the number of nodes belonging to the maximal sensing area $R\left(P_{t}\right)$. This probability is maximized for a period of sleep, $s$, such that: $s=2 \delta(n-1)-w$.

Given that the nodes of the network are mobile, we may also wish to maximize the probability, $p_{T}$, to discover a node during a given period of time $T$, if for example we are interested in the adjacencies that last at least $T$. This probability is defined as follows:

$$
P_{T}=1-\left[1-\frac{w-\delta}{w+s}\left(1-\frac{2 \delta}{w+s}\right)^{n-2}\right]^{\frac{T}{w+s}}
$$

where $\frac{T}{w+s}$ is the number of run during a period $T$. To analytically determine the theoretical value of $s$ which maximizes this probability is difficult. On the other hand, this can be numerically solved. 


\subsubsection{Energy consumption}

Knowing the frame size $F$ (equation 10), and the size of $s$, we can then deduce the various parameters of the hello protocol which give the lowest energy consumption in each node.

\subsection{Numerical example: the $C A P N E T$ project}

Within the context of the CAPNET project, it is planned to distribute sensors to the students of the french engineer school INSA de Lyon. The task of these sensors is the periodical discovery of the students neighborhood. The collected data will be useful to study graphs of interactions and students mobility. To reach this objective, it is necessary to tune the hello protocol in order to minimize the energy consumption and to detect the neighboring students up to a given distance.

In what follows, we present the characteristics of the sensors. Then, we describe the assumptions and the constraints we have in this scenario. Finally, we present the tuning of the hello protocol using the previous results.

\subsubsection{Description of the sensors}

The sensors which are used in the CAPNET's platform are built with the $C C 1100$ communication chipset of chipcon [2]. The $C C 1100$ is a RF transceiver characterized by a low power consumption and effective radio performances. The main characteristics of this transceiver are presented in table 1 .

\begin{tabular}{|c|c|}
\hline Transmission power (dbm) & from -30 to 10 \\
\hline Sensivity (dbm) & from -88 to -110 \\
\hline Frequency $(\mathrm{Mhz})$ & $400 / 800 / 900$ \\
\hline Data rate (kbps) & from 1,2 to 500 \\
\hline TX energy consumption (mA) & from 10 to 29 \\
\hline RX energy consumption $(\mathrm{mA})$ & from 14,2 to 15,4 \\
\hline
\end{tabular}

Table 1: CC1100 characteristics [2].

\subsubsection{Assumptions and constraints}

We consider the different available powers of transmission (from -30 to $10 \mathrm{dbm}$ ) with a sensivity threshold equal to $-88 \mathrm{dbm}$ and a frequency of $900 \mathrm{Mhz}$. We suppose a density of 5 nodes per $20 \mathrm{~m}^{2}$. We obtain then a density $\lambda$, such that: $\lambda=0.0125$.

We assume that the size of the hello packet is 18 bytes, and that the data rate of the sensors is $2.4 \mathrm{kbps}$. The transmission of one hello packet takes then a duration of $\delta=60 \mathrm{~ms}$.

Given that nodes ,i.e., the students, are mobile, we wish to discover adjacencies between students that last for a duration of at least one minute.

\begin{tabular}{|c|c|c|c|c|c|}
\hline$P_{t}(\mathrm{dbm})$ & -30 & -20 & -10 & 0 & 10 \\
\hline$R\left(P_{t}\right)(\mathrm{m})$ & 7,63 & 16,44 & 35,41 & 76,29 & 164,35 \\
\hline Number of nodes & 2,28 & 10,6 & 49,21 & 228,44 & 1060,17 \\
\hline TX $(\mathrm{mA})$ & 10 & 12 & 14 & 16 & 30 \\
\hline
\end{tabular}

Table 2: Sensors parameters.

In table 2 , we present the maximal sensing area, $R\left(P_{t}\right)$, associated to the various available transmission powers. The average number of nodes present in the disc of radius $R\left(P_{t}\right)$ and the energy consumption for the transmit mode are also presented. We assume that the average energy consumption in receive mode is $14,8 m A$.

Finally, we wish to tune the hello protocol to guarantee the discovery of the 4 closest neighbors. That is, we want that our protocol discovers all the nodes which are below a distance $L$, such that $L=10 \mathrm{~m}$. We thus use a transmission power superior or equal to $-20 \mathrm{dbm}$.

\subsubsection{Hello protocols' dimensioning}

To tune the hello protocol, we start with the computation of the maximal size of the frame $F$, (equation 10) allowing the discovery of all the nodes which are below a distance $L=10 \mathrm{~m}$. These sizes of $F$ are presented in table 3 .

\begin{tabular}{|c|c|c|c|c|}
\hline$P_{t}(\mathrm{dbm})$ & -20 & -10 & 0 & 10 \\
\hline maximal F (ms) & 1852 & 681 & 310 & 144 \\
\hline
\end{tabular}

Table 3: Maximal size of $F$.

We notice that for transmission powers larger or equal to $-10 \mathrm{dbm}$, the average number of nodes in the sensing area, $R\left(P_{t}\right)$, is raised. Furthermore, the maximal size of the frame $F$ is not rather sufficient to discover the nodes of the neighborhood. The probability of discovering a node, given by equation 5 , is too close to zero. Thus, these powers are not adapted to our needs. As a consequence, we use the following transmission power: $P_{1}=-20 \mathrm{dbm}$.

To compute the size of $s$ which maximizes the probability of discovering a node and minimizes the energy consumption, we resolve numerically the equation 12 . We fix the value of $w$ and we seek the optimal size of $s$ which maximizes equation 12 . The energy consumption is then estimated for each computed $s$.

In figure 9, according to the value of $w$, the optimal size of $s$ is shown. In figure 10, the resulting energy consumption is presented. Finally, the probability of discovering a node during a period of one minute is shown on figure 11 .

We notice that the more the size of $s$ decreases, the more the consumption of energy and the probability of discovering a node increase. Furthermore, we notice that the probability of discovering a node increases rather quickly, according to the size of $w$.

So, a configuration that maximizes the efficiency of our hello protocol could be the following one:

- An active period, such as: $w=180 \mathrm{~ms}$.

- A period of sleep, such as: $s=460 \mathrm{~ms}$.

- A frame F, such as: $F=640 \mathrm{~ms}$, which leads to an average number of runs of 94 per minute.

- An average energy consumption per minute of $234 \mathrm{~mA}$.

- A probability of discovering a node during one minute $p_{T}$, such as: $p_{T}=0.95$.

\section{CONCLUSIONS AND FUTURE WORK}

In this paper, we analyzed the problem of node discovery in large scale wireless multi-hop networks. We proposed a hybrid model allowing to estimate the real size of a sensing area in the presence of interferences. We showed by simulations the efficiency of this model for the prediction of the number of discovered nodes and the average distance to the most remote discovered node. Finally, we showed how to 
use the proposed model to tune a hello protocol. A real scenario stemming from the CAPNET project was proposed and analyzed as a numerical application.

Several perspectives remain open to investigations. We want to adapt our analysis to a probabilistic radio propagation model instead of the deterministic one that is currently used. We also want to extend our analytical study to consider variable parameters, such as the transmission power or the hello message frequency, for the different nodes. Finally, to cope with nodes' mobility, we wish to develop smart hello protocols, where nodes can adapt these parameters depending on the configuration of the network.

\section{REFERENCES}

[1] N. Abramson. The aloha system - another alternative for computer communication. In Proceedings of AFIPS, 1970.

[2] CC1100, 2006. http://www.chipcon.com.

[3] I. D. Chakeres and E. M. Belding-Royer. The utility of hello messages for determining link connectivity. In Proceedings of the 5th International Symposium on Wireless Personal Multimedia Communications (WPMC '02), 2:504-508, 27-30 Oct 2002.

[4] G. Chelius. The netsens simulator, 2006. http://worldsens.citi.insa-lyon.fr.

[5] B. B. F. Bacceli and P. Muhlethaler. An aloha protocol for multihop mobile wireless networks. In Proceedings of 16th ITC Specialist Seminar, 2004.

[6] H. Friis. A note on a simple transmission formula. In Proceedings of IRE, 1946.

[7] C. S. G. Alonso, E. Kranakis and P. Widmayer. Randomized protocols for node discovery in ad-hoc multichannel broadcast networks. In Proceedings of 2nd Annual Conference on Adhoc Networks and Wireless (ADHOCNOW'03), 2865:104-115, Oct 09-10 2003.

[8] R. W. G. Alonso, E. Kranakis and P. Widmayer. Probabilistic protocols for node discovery in ad-hoc, single broadcast channel networks (extended abstract). International Parallel and Distributed Processing Symposium (IPDPS'03), 2003.

[9] W. L. G. Jakllari and S. V. Krishnamurthy. An integrated neighbor discovery and mac protocol for ad hoc networks using directional antennas. In Proceedings of IEEE WoWMoM, 2005.

[10] V. C. Giruka and M. Singhal. Hello protocols for ad-hoc networks: Overhead and accuracy tradeoffs. Sixth IEEE International Symposium on a World of Wireless Mobile and Multimedia Networks (WoWMoM'05), June 13-16 2005.

[11] P. Gupta and P. Kumar. Capacity of wireless networks. IEEE Transactions on Information Theory, 46(2):388-404, 2000.

[12] H. Koskinen and J. Virtamo. Probability of successful transmission in a random slotted-aloha wireless multihop network employing constant transmission power. In Proceedings of the 8th ACM international symposium on Modeling, analysis and simulation of wireless and mobile systems, pages 191-199, 2005.

[13] M. J. McGlynn and S. A. Borbash. Birthday protocols for low energy deployment and flexible neighbor discovery in ad hoc wireless networks. In Proceedings of the 2nd ACM international symposium on Mobile ad hoc networking and computing (MobiHoc'01), pages 137-145, 2001.

[14] F. B. O. Dousse and P. Thiran. Impact of interferences on connectivity in ad hoc networks. In Proceedings IEEE INFOCOM, 2003.

[15] M. Y. S. Asami and K. Kagoshima. Differential hello technique for multihop wireless network routing protocols in dense environnements. IEICE TRANS. COMMUN., E88-B(1):292-303, 2005.

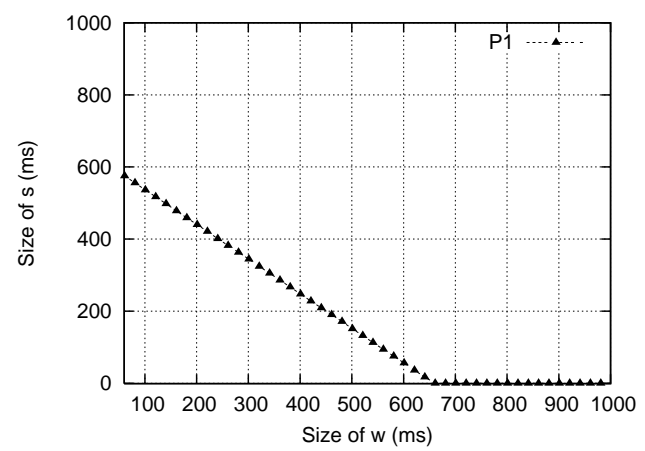

Figure 9: The optimal size of $s$.

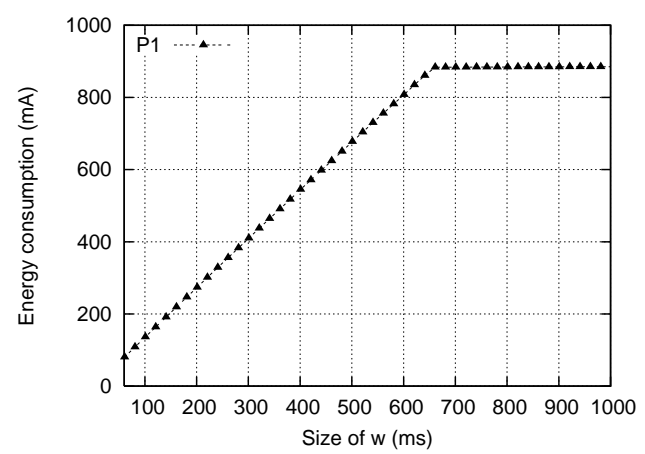

Figure 10: Energy consumption.

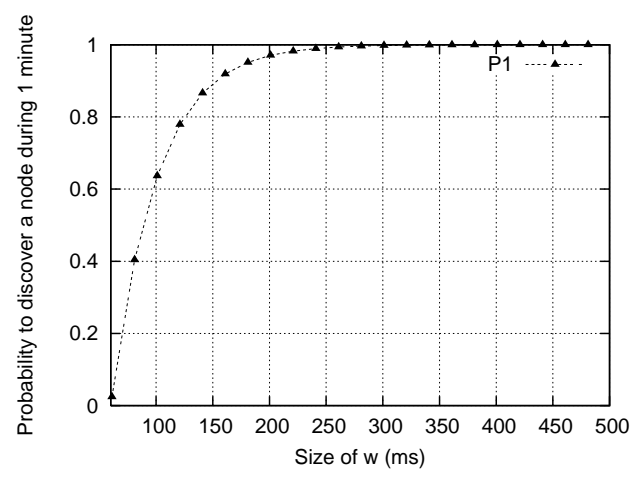

Figure 11: Probability to discover a node. 